\title{
Physical implications of a fundamental period of time
}

\author{
Garrett Wendel * Luis Martínez $\dagger$ and Martin Bojowald \\ Department of Physics, The Pennsylvania State University, \\ 104 Davey Lab, University Park, PA 16802, USA
}

\begin{abstract}
If time is described by a fundamental process rather than a coordinate, it interacts with any physical system that evolves in time. The resulting dynamics is shown here to be consistent provided the fundamental period of the time system is sufficiently small. A strong upper bound $T_{\mathrm{C}}<10^{-33} \mathrm{~s}$ of the fundamental period of time, several orders of magnitude below any direct time measurement, is obtained from bounds on dynamical variations of the period of a system evolving in time.
\end{abstract}

Dimensional arguments are often used to suggest that time has a fundamental period, given by the Planck time $t_{\mathrm{P}}=\sqrt{\hbar G / c^{5}}=5.39 \times 10^{-44} \mathrm{~s}$ using the speed of light $c$, Newton's constant $G$, and Planck's constant $\hbar$. Resolving this time scale is far beyond currently available technology. Nevertheless, it may be possible to obtain indirect information about physics near this scale, much like Brownian motion helped to confirm the atomic nature of matter using light microscopy, able to resolve only distance scales much larger than the atomic size. In order to devise indirect measurements, a detailed physical model must be available to derive effects that could magnify the sensitivity of a direct measurement. Here, we show that quantum mechanics of a physical model of time, described not as a monotonic external parameter but rather as a dynamical and oscillating variable that can model a physical clock, reveals a surprising magnification effect. The resulting upper bound on a potential fundamental period of time is about ten orders of magnitude above the Planck time, but much closer than any direct measurement could provide.

Formulating quantum mechanics with a physical, oscillating time variable may at first sight seem in conflict with the requirement of unitarity, which implies that the evolution operator between two states, $\psi(0)$ and $\psi(t)$, is given by $\hat{U}(t)=\exp (-i \hat{H} t / \hbar)$ using the self-adjoint Hamiltonian $\hat{H}$ of the evolving system. If this condition must be maintained for all $t$, it is impossible to make sense of a dynamical, oscillating time variable which turns back to its initial value after each clock cycle while the system does not, in general, evolve back. Moreover, even during phases in which the expectation value of a physical, and therefore quantum, time variable changes monotonically, the variable should be subject to quantum fluctuations which do not have a preferred direction. These problems are especially acute in quantum gravity and quantum cosmology, two fields which aim to quantize generally relativistic systems in which there is no absolute time [1-3].

A proposal to formulate a meaningful notion of physical time goes back to an investigation by Dirac [4] that analyzed general properties of quantum constrained systems relevant for generally relativistic systems. Dirac briefly suggested a construction, now called deparame- terization, which, with hindsight, can be interpreted as a solution to the problem of quantum fluctuations of a physical time variable by showing that physical time requires constrained dynamics: If both time and the system of interest are quantized in an extended model that includes all relevant degrees of freedom, the energies of the time variable and the system have to be exactly balanced. Otherwise, a non-zero net energy would imply non-trivial evolution of the extended model in an external absolute time parameter, violating the assumption that time is described by an internal degree of freedom.

A specific example from cosmology is the Friedmann equation

$$
\left(\frac{1}{a} \frac{\mathrm{d} a}{\mathrm{~d} t}\right)^{2}=\frac{8 \pi G}{3 c^{2}} \rho
$$

for the scale factor $a>0$, with the energy density $\rho$ of matter. In canonical form, this equation can be rewritten as an energy-balancing constraint

$$
C=-H_{\text {matter }}(V)+\frac{6 \pi G}{c^{2}} V p_{V}^{2}=0
$$

where $V=a^{3}$ is the spatial volume and $p_{V}=$ $-c^{2} \dot{a} /(4 \pi G a)$ its momentum. By virtue of the constraint, the matter energy $H_{\text {matter }}(V)=V \rho$ always equals the gravitational contribution $6 \pi G V p_{V}^{2} / c^{2}$.

A common matter system in cosmological models is a scalar degree of freedom $\phi$ with momentum $p_{\phi}$ and energy density

$$
\rho=\frac{1}{2} \frac{p_{\phi}^{2}}{V^{2}}+W(\phi)
$$

where $W(\phi)$ is the scalar potential, such as $W(\phi)=$ $\frac{1}{2} m^{2} \phi^{2}$ for a scalar of mass $m$. It is then possible to describe the expansion of the universe by a function $V(\phi)$ that determines the volume with reference to the value of the scalar, rather than using a time coordinate not described by a physical subsystem. To derive $V(\phi)$ classically, one first writes Hamilton's equations of motion by interpreting the constraint $C$ as the total Hamiltonian, such as $\mathrm{d} \phi / \mathrm{d} \epsilon=\partial C / \partial p_{\phi}=p_{\phi} / V$, and then eliminates the auxiliary parameter $\epsilon$ from the solutions $\phi(\epsilon)$ and $V(\epsilon)$, also using solutions for the momenta. (Systematic 
expansions that do not require intermediate $\epsilon$-dependent functions have been derived in $[5,6]$ ).

Deparameterization, in cosmological models following the constructions of [7], assumes that the scalar used as time is massless and without self-interactions, $W(\phi)=0$. Hamilton's equations derived from the constraint $C$ then imply that $p_{\phi}$ is conserved, while the rate of change of $\phi$, as just derived, is proportional to $p_{\phi}$. As long as $p_{\phi} \neq 0$, such that there is in fact energy in the time variable, $\phi$ is always monotonic in $\epsilon$. The assumption of zero scalar potential therefore does not allow one to describe oscillating clocks, but it shows how standard evolution as in quantum mechanics can formally be recovered: If we solve the constraint for $p_{\phi}$, we can quantize the resulting equation $-p_{\phi}=\sqrt{12 \pi G} V\left|p_{V}\right| / c$ by a Schrödinger equation

$$
i \hbar \frac{\partial \psi}{\partial \phi}=\frac{\sqrt{3 \pi G}}{c}\left(\hat{V}\left|\hat{p}_{V}\right|+\left|\hat{p}_{V}\right| \hat{V}\right) \psi .
$$

On solutions of the constraint, therefore, quantum fluctuations of $\phi$ do not present an obstacle to unitary evolution because they are no longer independent of the system degrees of freedom [8 11].

However, deparameterization, in spite of its widespread use in quantum cosmology, is not a realistic description of a fundamental process underlying our measurements of time because it would require fine-tuned interactions that prevent one variable from oscillating. The description of an oscillating motion would require the presence of a background time, such as the gauge parameter $\epsilon$ used above. Referring to such a monotonic background parameter might seem to render our logic circular. However, our construction will make use of a more general definition of an oscillating variable as one that enters a basic Hamiltonian with a standard kinetic energy and a mass term or some (self-)interaction potential that is unbounded from above. If one were to solve such a system in a background time, one would obtain oscillating motion, but we will require only the stated condition on the generic functional dependence of a Hamiltonian.

While monotonic readings can be constructed in specifically designed clocks or calendars, accounting for the fine-tuning required for a monotonic time variable to emerge, they do not refer to fundamental variables that would appear in a basic Hamiltonian with some potential or interaction term. Our model below will, in fact, describe a construction that shows how a monotonic time variable $(\tau)$ can emerge from an oscillating fundamental variable $(\phi)$. For instance, if we include a mass term in the cosmological model, $W(\phi)=\frac{1}{2} m^{2} \phi^{2}, \phi$ is an oscillating variable, and $p_{\phi}$ is no longer constant on solutions of the constraint (2). The time variable $\phi$ has turning points whenever $p_{\phi}$ equals zero.

A procedure to formulate quantum evolution with respect to such an oscillating time variable has only recently been given [12]. We illustrate and evaluate this procedure for a more familiar system from quantum mechanics rather than quantum cosmology, using a constraint

$$
C^{\prime}=p_{\phi}^{2}+\lambda^{2} \phi^{2}-H(x, p)^{2}
$$

where $H(x, p)$ is the Hamiltonian of a standard system such as the harmonic oscillator. For $\lambda=0$, we can use deparameterization, such that the quantized solution $-p_{\phi}=H(x, p)$ of $C^{\prime}=0$ is equivalent to the Schrödinger equation of quantum mechanics. For $\lambda \neq 0$,

$$
-p_{\phi}=\sqrt{H^{2}-\lambda^{2} \phi^{2}}
$$

is set equal to a time-dependent Hamiltonian which becomes problematic if we try to interpret $\phi$ as a global time that can take any real number because $p_{\phi}$ would not always be real.

In order to address this problem, one first constructs a global time variable, $\tau$, such that $\phi(\tau)= \pm \tau+A$ with constant $A$ is linear in $\tau$, with unit rate, between any two turning points of $\phi$. Different phases of $\phi$, separated by turning points, are related by choosing constants $A$ in each phase such that $\phi(\tau)$ is continuous with alternating $\mathrm{d} \phi / \mathrm{d} \tau= \pm 1$. Without changing the dynamics, $\tau$ then provides a global, monotonically increasing time parameter that unravels the motion of $\phi$, just like standard clocks unfold the circular motion of the minute hand by moving forward the hour hand after one minute cycle has been completed. (A similar procedure has been applied to a related case in which non-monotonic behavior is a consequence of non-trivial topology rather than turning points [13, 14].) In the example of $C^{\prime}$, (5), we use

$$
\phi(\tau)=\left\{\begin{array}{cl}
(4 n+2) \phi_{\mathrm{t}}-\tau & \text { if } 4 n+1 \leq \tau / \phi_{\mathrm{t}} \leq 4 n+3 \\
\tau-4 n \phi_{\mathrm{t}} & \text { otherwise }
\end{array}\right.
$$

where $\phi_{\mathrm{t}}=H / \lambda$ characterizes turning points $\left(p_{\phi}=0\right)$. The integer

$$
n=\left\lfloor\frac{1+\tau / \phi_{\mathrm{t}}}{4}\right\rfloor
$$

determines the number of cycles of $\phi$ the fundamental clock goes through between time 0 and time $\tau$. Evaluating (6) in $\phi(\tau)$ always gives a real number, for any $\tau$.

In the second step, we formulate quantum evolution with respect to $\tau$ by concatenating evolution operators for monotonic phases of $\phi$. The Schrödinger equation implied by (6) can be solved in the energy eigenbasis $\psi_{k}$ of the system with Hamiltonian $\hat{H}$ with energy eigenvalues $E_{k}: \psi_{k}(q, \phi)=\psi_{k}(q, 0) \exp \left(i \Theta_{k}(\phi)\right)$ with the phase

$$
\Theta_{k}(\phi)=-\frac{1}{2 \lambda \hbar}\left(\lambda \phi \sqrt{E_{k}^{2}-\lambda^{2} \phi^{2}}+E_{k}^{2} \arcsin \left(\frac{\lambda \phi}{E_{k}}\right)\right) .
$$


This phase is real only for $\phi$ between its turning points, and therfore, as expected, $\phi$ does not provide global evolution. However, $\Theta_{k}(\phi(\tau))$ is always real and implies global evolution with respect to $\tau$. Because $\mathrm{d} \phi / \mathrm{d} \tau= \pm 1$ is not constant, however, we should alternate the sign of $\Theta_{k}(\phi(\tau))$ in order to describe evolution with respect to $\tau$ instead of $\phi$. (The correct equation is a slight modification of (4), changing $i \hbar \partial \psi / \partial \phi=\hat{H} \psi$ to $i \hbar \partial \psi / \partial \tau=$ $(\mathrm{d} \phi / \mathrm{d} \tau) \hat{H} \psi$.)

The construction just described introduces welldefined, unitary evolution with respect to $\tau$, implying a realistic model of time in which a system evolves relative to an oscillating quantum clock. The clock and the system are interacting through the energy-balance condition $C^{\prime}=0$, (5), which implies rather complicated time-dependent Hamiltonians for $\lambda \neq 0$. Numerical simulations can however be performed and reveal several interesting and surprising properties. In order to bring these out most clearly, we now specify the system Hamiltonian to be given by the harmonic oscillator, but the relevant features have been confirmed numerically also for anharmonic and atomic systems.

For the harmonic oscillator, we expect that the strong coherence of the standard system, realized for $\lambda=0$, disappears for $\lambda \neq 0$ in which case the quadratic Hamiltonian is replaced by (6). This expectation is confirmed in Fig. 1. However, the same figure shows that coherence remains intact for large $\lambda$, defined as values of $\lambda$ such that the clock period $T_{\mathrm{C}}=4 \phi_{\mathrm{t}}=4 H / \lambda$ is much smaller than the system period. When the clock goes through many cycles during a single system period, therefore, the dynamics is almost indistinguishable from what is known from standard quantum mechanics. The only visible difference is a system period rescaled by a factor of $\pi / 4$, which can always be absorbed in a redefinition of system parameters.

The rescaled period can be explained as follows: During each clock cycle, while $\phi$ runs from $-\phi_{\mathrm{t}}=-E_{k} / \lambda$ to $\phi_{\mathrm{t}}$ and back, the wave function accumulates a phase difference of

$$
\Delta \Theta_{k}=2\left(\Theta_{k}\left(\phi_{\mathrm{t}}\right)-\Theta_{k}\left(-\phi_{\mathrm{t}}\right)\right)=-\frac{\pi E_{k}^{2}}{\hbar} \frac{n}{\lambda}
$$

in each stationary state. Moreover, for large $\lambda$, the number of cycles, $n$ given in (8), divided by $\lambda$ can be approximated by

$$
\frac{n}{\lambda}=\frac{1}{4}\left\lfloor 1 / \lambda+\tau / E_{k}\right\rfloor \approx \frac{\tau}{4 E_{k}} .
$$

Therefore, the phase accumulated over many cycles is approximately given by

$$
\Delta \Theta_{k} \sim-\frac{\pi E_{k} \tau}{4 \hbar}
$$

which is $\pi / 4$ times the standard phase $-E_{k} t / \hbar$.
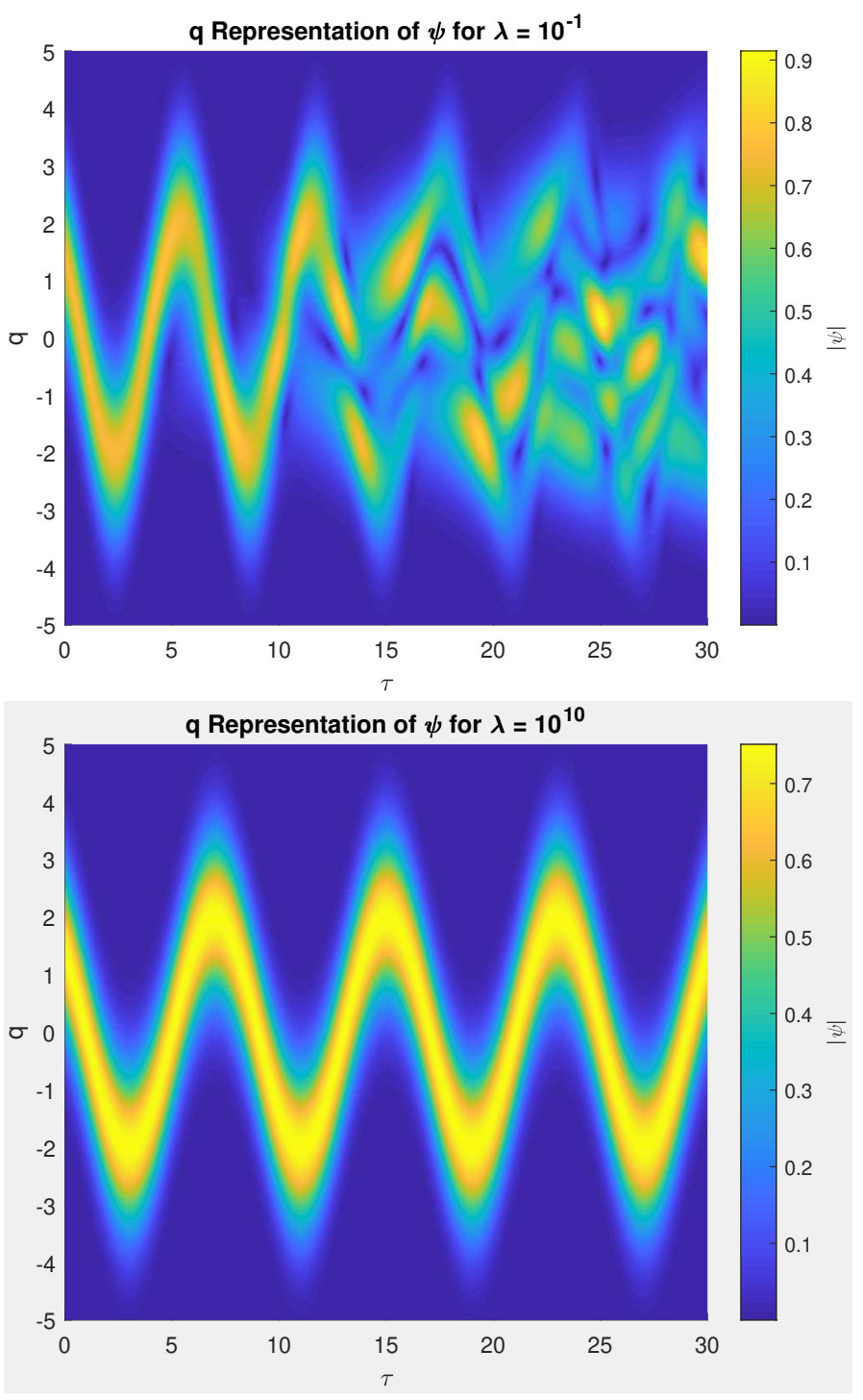

FIG. 1. Density plots of the wave function for small $\lambda$ (top) and large $\lambda$ (bottom), respectively, using a harmonicoscillator Hamiltonian $\hat{H}=\frac{1}{2}\left(\hat{p}^{2}+\hat{q}^{2}\right)$ and a coherent initial state.

Our first result is therefore an unexpected revival of coherence for small periods of a fundamental clock. Differences between deparameterization with a monotonic time variable and the realistic implementation of a physical and oscillating clock are tiny, providing justification for the deparameterization procedure as a simplified mathematical method that is nevertheless able to describe implications of an oscillating clock. Deparameterization, as envisioned by Dirac, is therefore viable as a procedure that allows one to understand qualitative features of relativistic quantum systems. (Other aspects of the problem of time are still being studied, mainly related to transforming observables obtained with different time or frame choices [15 20].) However, our second result, to be described in the remainder of this letter, shows that 


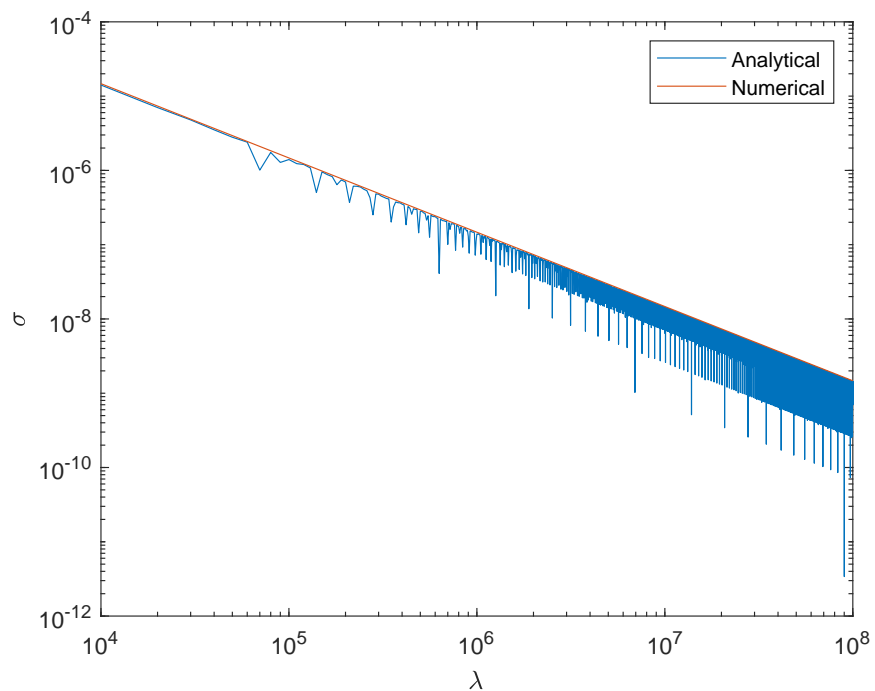

FIG. 2. Relative standard deviation $\sigma$ of the system period over many system cycles as a function of $\lambda$. The analytical approximation (13) agrees well with the upper limit of a numerical computation of many system periods, both confirming a $1 / \lambda$-behavior of $\sigma$.

there are small effects of a physical, periodic clock that can be relevant for sensitive observations.

Additional deviations from the standard behavior can be uncovered by detailed numerical analysis. In particular, because the phase (9) is not linear in $\tau$, the system does not go through its cycles in a uniform manner, as would be the case with the standard linear phase $-E_{k} t / \hbar$ for each stationary contribution. As a consequence, the distribution of system periods taken over large evolution times has a non-zero standard deviation, as shown in Fig. 2. Importantly, the plot shows a simple $1 / \lambda$ dependence of the standard deviation, which can be used in an extrapolation to periods that would be too small for accurate numerical evaluations.

Also the $1 / \lambda$-behavior can be derived analytically for large $\lambda$. We average the squared deviation of the phase (9) from the linear limit of $\lambda \rightarrow \infty, \Theta_{k}^{\infty}(\tau)=\Delta \Theta_{k}$ given in (12), over a half-cycle of $\phi$ :

$$
\begin{aligned}
\sigma^{2} & =\frac{1}{\phi_{\mathrm{t}}} \int_{0}^{\phi_{\mathrm{t}}}\left(\Theta_{k}(\phi(\tau))-\Theta_{k}^{\infty}(\tau)\right)^{2} \mathrm{~d} \tau \\
& =\frac{E_{k}^{4}\left(21 \pi^{2}-1024 / 5\right)}{24^{2} \lambda^{2} \hbar^{2}} .
\end{aligned}
$$

Therefore, the clock period $T_{\mathrm{C}}=4 \phi_{\mathrm{t}}=4 E_{k} / \lambda$ in a stationary state is related to the system period $T_{\mathrm{S}}=2 \pi \hbar / E_{k}$ by

$$
T_{\mathrm{C}}=\frac{48 \sigma T_{\mathrm{S}}}{\pi \sqrt{21 \pi^{2}-1024 / 5}} \approx 9.7 \sigma T_{\mathrm{S}}
$$

Before we evaluate this result, we note that the qualitative behavior is robust and does not depend much on
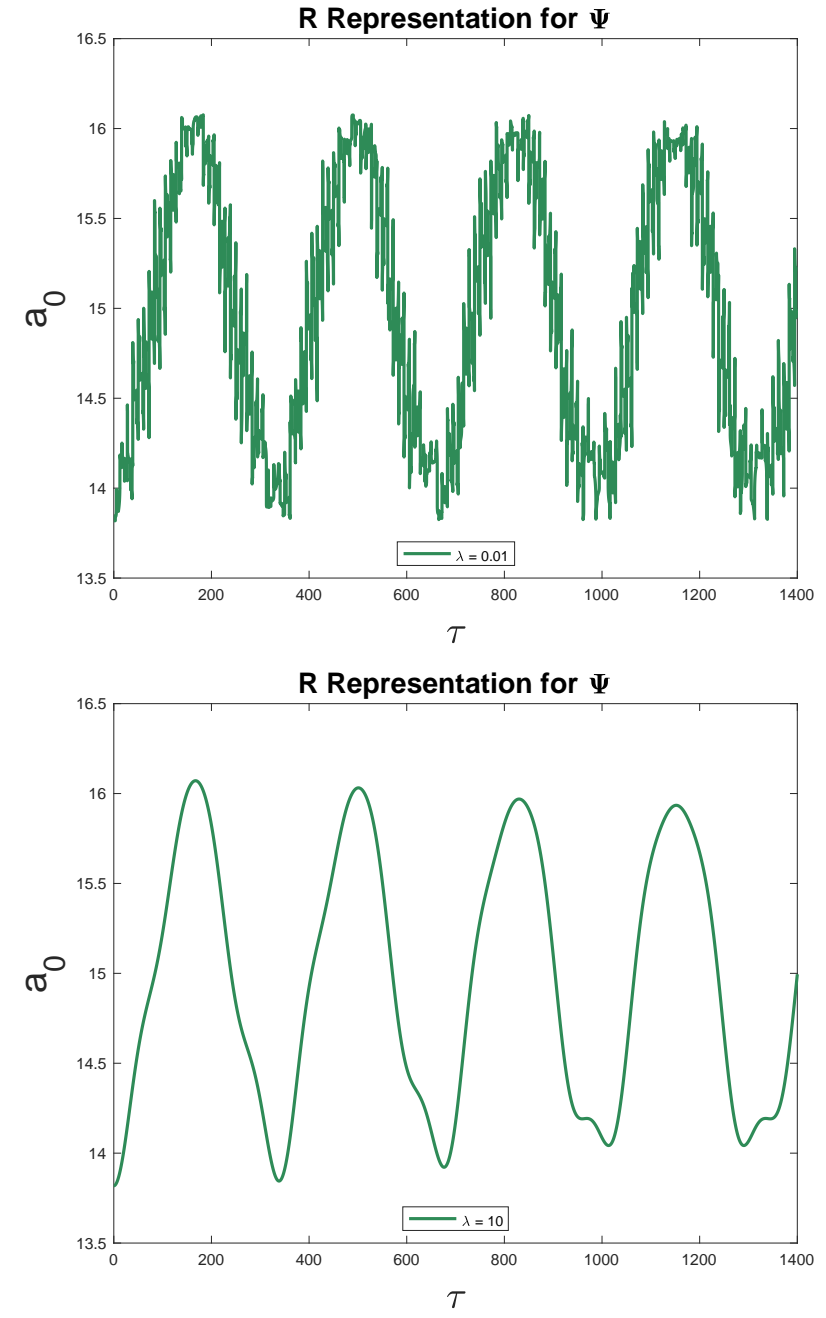

FIG. 3. Radius expectation values as multiples of the Bohr radius $a_{0}$ for small $\lambda$ (top) and large $\lambda$ (bottom), respectively, using a superposition of hydrogen eigenstates. The example of large $\lambda$ is visually indistinguishable from $\lambda=0$.

the precise dynamics of the fundamental clock. For a clock Hamiltonian other than $p_{\phi}^{2}+\lambda^{2} \phi^{2}$, the phase $\Theta_{k}$ would be different, implying changes in the scaling factor of $\pi / 4$ in the system period and in the coefficients of (14). However, results analogous to our specific equations would still be obtained. As long as we are interested in an upper bound on the fundamental period of time, therefore, the clock details do not matter. Considering systems other than a harmonic oscillator, for the same fundamental clock, does not change our results because we referred only to generic $E_{k}$ and $\psi_{k}$. They may be more difficult to obtain for non-harmonic systems, but their specific form is not required for our equations such as (13). A non-harmonic example is shown in Fig. 3.

Given the relative precision $\sigma$ of a time measurement, such as $\sigma \approx 10^{-19}$ for recent atomic clocks [21] working at a system period of $T_{\mathrm{S}} \approx 2 \mathrm{fs}$ (corresponding to the wave 
length $698 \mathrm{~nm}$ of the ${ }^{3} P_{0} \rightarrow{ }^{1} S_{0}$ transition of Strontium), we therefore obtain the upper bound $T_{\mathrm{C}}<10^{-33} \mathrm{~s}$ : The measured precision could not be maintained if $T_{\mathrm{C}}$ were greater, implying a non-uniform system period. This upper bound is about ten orders of magnitude above the Planck time, but it is much smaller than could be achieved with any direct measurement.

Acknowledgements: This work was supported in part by NSF grant PHY-1912168. LM was supported by a Gates Scholarship.

* gmw5164@psu.edu

† lxm471@psu.edu

$\ddagger$ bojowald@gravity.psu.edu

[1] K. V. Kuchař, in Proceedings of the 4th Canadian Conference on General Relativity and Relativistic Astrophysics, edited by G. Kunstatter, D. E. Vincent, and J. G. Williams (World Scientific, Singapore, 1992).

[2] C. J. Isham, Integrable systems, quantum groups, and quantum field theory (Kluwer, Dordrecht, 1993), pp. 157287.

[3] E. Anderson, in Classical and Quantum Gravity: Theory, Analysis and Applications, edited by V. R. Frignanni (Nova, New York, 2012).

[4] P. A. M. Dirac, Can. J. Math. 2, 129 (1950).

[5] B. Dittrich, Gen. Rel. Grav. 39, 1891 (2007), gr-qc/0411013
[6] B. Dittrich, Class. Quant. Grav. 23, 6155 (2006), gr-qc/0507106.

[7] W. F. Blyth and C. J. Isham, Phys. Rev. D 11, 768 (1975).

[8] M. Bojowald, P. A. Höhn, and A. Tsobanjan, Class. Quantum Grav. 28, 035006 (2011), arXiv:1009.5953.

[9] M. Bojowald, P. A. Höhn, and A. Tsobanjan, Phys. Rev. D 83, 125023 (2011), arXiv:1011.3040.

[10] P. A. Höhn, E. Kubalova, and A. Tsobanjan, Phys. Rev. D 86, 065014 (2012), arXiv:1111.5193.

[11] M. Bojowald and A. Tsobanjan, arXiv:1906.04792.

[12] M. M. Amaral and M. Bojowald, Ann. Phys. 388C, 241 (2018), arXiv:1601.07477.

[13] B. Dittrich, P. A. Hoehn, T. A. Koslowski, and M. I. Nelson, arXiv:1508.01947.

[14] B. Dittrich, P. A. Hoehn, T. A. Koslowski, and M. I. Nelson, Phys. Lett. B 769, 554 (2017), arXiv:1602.03237.

[15] P. Malkiewicz, Class. Quantum Grav. 29, 075008 (2012), arXiv:1105.6030

[16] P. Malkiewicz, Class. Quantum Grav. 32, 135004 (2015), arXiv:1407.3457

[17] F. Giacomini, A. Castro-Ruiz, and C. Brukner, Nat. Commun. 10, 494 (2019), arXiv:1712.07207.

[18] A. Vanrietvelde, P. A. Hoehn, F. Giacomini, and E. Castro-Ruiz, Quantum 4, 225 (2020), arXiv:1809.00556.

[19] A. Vanrietvelde, P. A. Hoehn, and F. Giacomini, arXiv:1809.05093.

[20] P. A. Hoehn, A. R. H. Smith, and M. P. E. Lock, arXiv:1912.00033.

[21] S. L. Campbell et al., Science 358, 90 (2017). 